\title{
Role of KIF2A in the progression and metastasis of human glioma
}

\author{
XIN ZHANG $^{1,2}$, CHAO MA $^{1}$, QINGJIE WANG ${ }^{1}$, JIA LIU $^{1}$, MINGYI TIAN $^{1}$, \\ YUCHANG YUAN $^{1}, \mathrm{XINGANG} \mathrm{LI}^{3}$ and XUN QU ${ }^{1}$ \\ ${ }^{1}$ Institute of Basic Medical Sciences, Qilu Hospital of Shandong University, Jinan, Shandong 250012; \\ ${ }^{2}$ Department of Oncology, The First Affiliated Hospital of Liaoning Medical University, Jinzhou, Liaoning 121000; \\ ${ }^{3}$ Department of Neurosurgery, Qilu Hospital of Shandong University and Brain Science Research Institute, \\ Shandong University, Jinan, Shandong 250012, P.R. China
}

Received February 6, 2015; Accepted November 25, 2015

DOI: $10.3892 / \mathrm{mmr} .2015 .4700$

\begin{abstract}
KIF2A is a member of the kinesin- 13 family and is involved in cell mitosis and spindle assembly. The abnormal expression and dysfunction of KIF2A are associated with tumorigenesis and the progression of certain human cancer types; however, little is known about the expression and roles of KIF2A in glioma. In the present study, the expression of KIF2A in 35 freshly isolated human glioma tissue samples was analyzed using reverse transcription-quantitative polymerase chain reaction (RT-qPCR) and immunohistochemistry (IHC). The association between the expression of KIF2A and clinicopathological parameters were assessed using the $\chi^{2}$ test. The biological functions of KIF2A on the proliferation, apoptosis, migration and invasion of glioma cells were investigated using an A172 cell line. RT-qPCR and IHC results demonstrated that the mRNA and protein expression levels of KIF2A were significantly higher in grade III-IV glioma tissues compared with those in grade I-II glioma tissues $(\mathrm{P}<0.05)$. No significant correlation was observed between the expression of KIF2A, and age, gender, tumor location and size $(\mathrm{P}>0.05)$. In vitro, KIF2A gene silencing inhibited the proliferation, migration and invasion, and induced apoptosis in cancer cells. The present data suggested that KIF2A may be important in glioma progression and may serve as a novel predictive factor and therapeutic target for glioma.
\end{abstract}

\section{Introduction}

Glioma is the most common tumor type of the central nervous system and they occur in 6.42/100,000 individuals (1). Despite the advancements in surgery, radiotherapy and chemotherapy, the survival rate of patients with high grade glioma has not

Correspondence to: Professor Xun Qu, Institute of Basic Medical Sciences, Qilu Hospital of Shandong University, 107 Wenhuaxi Road, Jinan, Shandong 250012, P.R. China

E-mail: quxun@sdu.edu.cn

Key words: glioma, KIF2A, migration/invasion, growth significantly improved during the past several decades, predominantly due to regional recurrence (2).

The kinesin superfamily proteins (KIFs) are a conserved class of microtubule-dependent molecular motor proteins exhibiting adenosine triphosphatase activity. KIFs are important in mitosis, meiosis and macromolecular transport (3). The abnormal expression and dysfunction of KIFs may lead to the development and progression of various types of human cancer (4-8). KIF2A, a member of the kinesin-13 family, is known to be involved in mitosis and mitotic spindle assembly (9). Previously, the expression of KIF2A was demonstrated to be upregulated in squamous cell carcinoma of the oral tongue (SCCOT) and breast cancer, when compared with the adjacent tissues $(10,11)$. KIF2A promotes SCCOT progression and metastasis, and breast cancer metastasis; however, little is known about the expression and roles of KIF2A in glioma.

In the present study, the expression of KIF2A was examined in 35 freshly isolated human glioma tissue samples, and its prognostic value for glioma patients was evaluated. The biological functions of KIF2A in glioma cells were also analyzed.

\section{Materials and methods}

Patient specimens. Intracranial tissue specimens were collected from 35 patients [ 23 males $(65.7 \%)$ and 12 females (34.3\%)] and included 15 grade I-II cases (42.9\%) and 20 grade III-IV cases (57.1\%), ranging in age from 4 to 70 years. The patients underwent primary and curative resection for glioma, and were pathologically confirmed at Qilu Hospital of Shandong University (Jinan, China) in 2014, for reverse transcription quantitative-polymerase chain reaction (RT-qPCR) and immunohistochemical (IHC) analyses. The patients were diagnosed based on the World Health Organization (2007) standard classification (12). The fresh tumor tissues were immediately transferred to liquid nitrogen and stored at $-80^{\circ} \mathrm{C}$ for subsequent RT-qPCR. The remaining tissue was immediately dipped in 10\% formalin (Sangon Biotech Co., Ltd., Shanghai, China) for IHC analysis. The present study was approved by the Institutional Review Board of Qilu Hospital of Shandong University, and written informed consent was obtained from each patient or the patient's family. 
$R T-q P C R$. The total RNA was extracted from human intracranial tissues or cells using TRIzol reagent (Invitrogen; Thermo Fisher Scientific, Inc., Waltham, MA, USA). Single-stranded cDNA was synthesized using oligo dT16 primers and Moloney Murine Leukemia Virus Reverse Transcriptase, according to the manufacturer's protocol (Invitrogen; Thermo Fisher Scientific, Inc.). RT-qPCR was performed using a LightCycler 2.0 Instrument (Roche Diagnostics $\mathrm{GmbH}$, Penzberg, Germany) and the cycling conditions were as follows: $30 \mathrm{sec}$ at $95^{\circ} \mathrm{C}$, and 40 cycles at $95^{\circ} \mathrm{C}$ for $5 \mathrm{sec}, 60^{\circ} \mathrm{C}$ for $10 \mathrm{sec}$ and $72^{\circ} \mathrm{C}$ for $15 \mathrm{sec}$ for PCR amplification, according to the instructions of the SYBR ${ }^{\circledR}$ Green Realtime PCR Master Mix [Toyobo (Shanghai) Co., Ltd.], which was used as the detection dye. Gene-specific amplifications were confirmed through a melting curve analysis at the end of the RT-qPCR. Relative gene expression levels were determined using the $2^{-\Delta \Delta \mathrm{Cq}}$ method with endogenous glyceraldehyde-3-phosphate dehydrogenase (GAPDH) used as the reference gene. The following primers were used: KIF2A, sense: 5'-GCCTTTGAT GACTCAGCTCC-3' and antisense: 5'-TTCCTGAAAAGT CACCACCC-3' (154 bp); GAPDH, sense: 5'-GGTGGTCTC CTCTGACTTCAACAG-3' and antisense: 5'-GTTGCTGTA GCCAAATTCGTTGT (127 bp).

IHC analysis and evaluation of immunostaining parameters. The IHC procedure was performed according to an established protocol, with minor modifications (13). Briefly, all glioma tissues were formalin-fixed, paraffin-embedded (Sangon Biotech Co., Ltd.), sectioned at $4 \mu \mathrm{m}$ thickness using a rotatory microtome (Finesse ${ }^{\mathrm{TM}}$ 325; Thermo Shandon Ltd., Cheshire, UK) and then placed on slides pretreated with 3-aminopropyltriethoxysilane (ZSGB-Bio, Beijing, China). Following deparaffinization and rehydration, the sections were microwaved for heat-induced epitope retrieval in citrate buffer (ZSGB-Bio; $80^{\circ} \mathrm{C}$ ). The sections were subsequently washed with phosphate-buffered saline (PBS) and the endogenous peroxidase activity was inhibited with $3 \%$ hydrogen peroxide for $10 \mathrm{~min}$. Next, the specimens were blocked with PBS containing normal sheep serum (ZSGB-Bio) at $37^{\circ} \mathrm{C}$ for $30 \mathrm{~min}$. Subsequently, the sections were incubated with rabbit anti-human anti-KIF2A antibody $(1: 4,000$; Abcam, Cambridge, UK; cat. no. ab37005) overnight at $4^{\circ} \mathrm{C}$ in a humidified chamber. Following rinsing with PBS, the slides were incubated for $30 \mathrm{~min}$ with secondary anti-rabbit antibody conjugated with horseradish peroxidase, according to the manufacturer's instructions for the PV-9000 2-step plus ${ }^{\circledR}$ Poly-HRP Anti-Mouse/Rabbit IgG Detection System kit (ZSGB-Bio; 1:100). The slides were then exposed to diaminobenzidine for visualization and hematoxylin (ZSGB-Bio) for nuclear counterstaining.

The tissue sections were assessed by light microscopy (Olympus IX51; Olympus Corp., Tokyo, Japan; magnification, x200) by two pathologists in a blinded manner. Initially, a proportion score was assigned, representing the estimated proportion of positive tumor cells ( 0 , none; $1,1 / 100$; $2,1 / 100-1 / 10 ; 3,1 / 10-1 / 3 ; 4,1 / 3-2 / 3 ; 5,>2 / 3)$. Next, an intensity score was assigned, representing the average intensity of the positive tumor cells $(0$, none; 1 , weak; 2 , intermediate; 3 , strong). The proportion and intensity scores were subsequently added to obtain a total score ranging between 0 and 8 , with 0-3 indicating negative and 4-8 indicating positive (14). The specimens were rescored if the difference between the scores from the two pathologists was $>3$.

Western blot analysis. Cultured cells $\left(4 \times 10^{5}\right)$ in a 6 -well cell plate were homogenized for $60 \mathrm{~min}$ in $150 \mu \mathrm{l}$ ice-cold radioimmunoprecipitation lysis buffer $[50 \mathrm{mM}$ Tris- $\mathrm{HCl}$ (pH 7.4), $150 \mathrm{mM} \mathrm{NaCl,1 \%}$ Triton $\mathrm{X}-100,1 \%$ sodium deoxycholate, $0.1 \%$ SDS, $2 \mathrm{mM}$ sodium pyrophosphate, $25 \mathrm{mM}$ $\beta$-glycerophosphate, $1 \mathrm{mM}$ EDTA, $1 \mathrm{mM} \mathrm{Na}_{3} \mathrm{VO}_{4}$ and $0.5 \mathrm{mg} / \mathrm{ml}$ leupeptin] according to the manufacturer's instructions (cat. no. P0013K; Beyotime Institute of Biotechnology, Beijing, China), containing $1 \mathrm{mM}$ phenylmethylsulfonyl fluoride (Beyotime Institute of Biotechnology). The samples were subsequently centrifuged at $15,000 \mathrm{x}$ g for $10 \mathrm{~min}$ at $4^{\circ} \mathrm{C}$. The protein concentration was measured using a Bicinchoninic acid Protein Assay kit (Beyotime Institute of Biotechnology). The protein samples were denatured by boiling for $10 \mathrm{~min}$ prior to electrophoresis. A total of $10 \mu \mathrm{g}$ each protein sample was separated by $10 \%$ sodium dodecyl sulfate polyacrylamide gel electrophoresis (SDS-PAGE) and were subsequently transferred onto a polyvinylidene fluoride membrane (EMD Millipore, Darmstadt, Germany) at $100 \mathrm{~V}$ for $90 \mathrm{~min}$. Following blocking with 5\% non-fat milk for $1 \mathrm{~h}$ at room temperature, each membrane was incubated with primary antibodies, including rabbit polyclonal anti-KIF2A (1:4,000), rabbit polyclonal anti-MMP-2 (1:1,000; Abcam; cat. no. ab110186), rabbit polyclonal anti-MMP-9 (1:1,000; Cell Signaling Technology, Danvers, MA, USA; cat. no. 3852) or rabbit anti-GAPDH polyclonal GAPDH (1:2,000; Proteintech Group, Inc., Wuhan, China; cat. no. 10494-1-AP), overnight at $4^{\circ} \mathrm{C}$, followed by three $10 \mathrm{~min}$ washes with Tris-buffered saline, containing $0.1 \%$ Tween-20 (TBST). Following incubation with peroxidase-conjugated affinipure goat anti-rabbit IgG(H+L) (1:5,000; Santa Cruz Biotechnology Inc., Santa Cruz, CA, USA; cat. no. SA00001-2) for $1 \mathrm{~h}$ at room temperature, the membranes were washed three times with TBST for $10 \mathrm{~min}$. The bands were subsequently detected by enhanced chemiluminescence (EMD Millipore). The housekeeping protein GAPDH served as a loading control. Positive immunoreactive bands and the ratio of target proteins to GAPDH in optical density units were obtained using Image Station 4000 MM software (Carestream Health, Rochester, NY, USA).

Cell culture and RNA interference. The human malignant glioma cell lines, A172 and U251 were obtained from the Cell Bank of Type Culture Collection of the Chinese Academy of Sciences (Shanghai, China). The cells were cultured in Dulbecco's modified Eagle's medium (DMEM; Hyclone; Thermo Fisher Scientific, Inc.), supplemented with $10 \%$ fetal bovine serum (FBS; Gibco; Thermo Fisher Scientific, Inc.) at $37^{\circ} \mathrm{C}$ in a $5 \% \mathrm{CO}_{2}$ humidified incubator. For RNA interference, the A172 cells were transfected with 50 nM KIF2A small interfering (si)RNA (5'-CACCGGCAAAGAGAT TGACCTGGTTCAAGAGACCAGGTCAATCTCTTTGCC TTTTTTG-3') or scrambled siRNA (a universal negative control; 5'-CACCGTTCTCCGAACGTGTCACGTCAAGAG ATTACGTGACACGTTCGGAGAATTTTTTG-3') using Lipofectamine 2000 (Invitrogen; Thermo Fisher Scientific, Inc.), according to the manufacturer's protocol. 
Table I. mRNA and protein expression levels of KIF2A and its association with patient clinicopathological features.

\begin{tabular}{|c|c|c|c|c|c|c|c|}
\hline \multirow[b]{2}{*}{ Variable } & \multirow[b]{2}{*}{ Patients, $\mathrm{n}$} & \multicolumn{3}{|c|}{ mRNA expression ${ }^{a}$} & \multicolumn{3}{|c|}{ Protein expression, $\mathrm{n}(\%)$} \\
\hline & & Median & IQR & P-value & Positive & Negative & P-value \\
\hline Age, years & & & & $0.056^{\mathrm{b}}$ & & & $0.317^{\mathrm{d}}$ \\
\hline$\leq 45$ & 14 & 0.009 & $0.005-0.020$ & & $6(17.14)$ & $8(22.88)$ & \\
\hline$>45$ & 21 & 0.020 & $0.010-0.050$ & & $13(37.14)$ & $8(22.88)$ & \\
\hline Gender & & & & $0.263^{\mathrm{b}}$ & & & $1.000^{\mathrm{d}}$ \\
\hline Male & 23 & 0.020 & $0.008-0.050$ & & $12(34.29)$ & $11(31.43)$ & \\
\hline Female & 12 & 0.020 & $0.004-0.028$ & & $7(20.00)$ & $5(14.29)$ & \\
\hline Tumor location & & & & $0.143^{\mathrm{c}}$ & & & $0.541^{\mathrm{d}}$ \\
\hline Prefrontal & 14 & 0.020 & $0.005-0.050$ & & $6(17.14)$ & $8(22.88)$ & \\
\hline Temporal lobe & 13 & 0.020 & $0.009-0.055$ & & $8(22.88)$ & $5(14.29)$ & \\
\hline Other & 8 & 0.090 & $0.039-0.175$ & & $5(14.29)$ & $3(8.57)$ & \\
\hline Grade & & & & $0.033^{\mathrm{b}}$ & & & $0.044^{\mathrm{d}}$ \\
\hline I-II & 15 & 0.009 & $0.005-0.020$ & & $5(14.29)$ & $10(28.57)$ & \\
\hline III-IV & 20 & 0.025 & $0.010-0.073$ & & $14(40.00)$ & $6(17.14)$ & \\
\hline Tumor size, $\mathrm{cm}$ & & & & $0.601^{\mathrm{b}}$ & & & $0.503^{\mathrm{d}}$ \\
\hline$\geq 3$ & 22 & 0.020 & $0.008-0.050$ & & $13(37.14)$ & $9(25.71)$ & \\
\hline$<3$ & 13 & 0.010 & $0.007-0.030$ & & $6(17.14)$ & $7(20.00)$ & \\
\hline
\end{tabular}

${ }^{\mathrm{a}}$ Continuous value calculated from the ratio of KIF2A and GAPDH; ${ }^{\mathrm{b}} \mathrm{Mann}-$ Whitney $\mathrm{U}$ test; ${ }^{\mathrm{c}} \mathrm{Kruskal}-$ Wallis test; ${ }^{\mathrm{d}} \chi^{2}$ test. P<0.05 was considered to indicate a statistically significant difference. The median represents the 50 th percentile of the mRNA expression level. The median and $\mathrm{IQR}$ were used to describe the mRNA expression level, as the mRNA expression level distribution is non-normal. IQR, inter-quartile range.

Cell proliferation assay. A172 cells were transfected with either KIF2A siRNA or scrambled siRNA and seeded at a density of $1.0 \times 10^{4}$ cells/well in a 96 -well plate. Following incubation for $0,24,48$ and $72 \mathrm{~h}$, a CCK-8 assay (Yiyuan Biotech Inc., Guangzhou, China) was performed to measure cell proliferation, according to the manufacturer's protocol. The optical densities were measured at $450 \mathrm{~nm}$ using a TECAN Infinite ${ }^{\circledR}$ M200 microplate reader (Tecan US, Durham, NC, USA).

Matrigel invasion and Transwell migration assays. The effects of KIF2A on the invasion and migration of glioma cells were evaluated using a Matrigel invasion and a transwell migration assay in a 24-well Transwell, containing polycarbonate filters with $8 \mathrm{~mm}$ pores (Costar, Corning, NY, USA). For the Matrigel cell invasion assay, the inserts were precoated with $50 \mu 1$ Matrigel matrix (dilution at 1:3; BD Bioscience, Franklin Lakes, NJ, USA), according to the manufacturer's protocol. At $48 \mathrm{~h}$ after the transfection with KIF2A siRNA or scrambled siRNA, the A172 cells were trypsinized (Invitrogen; Thermo Fisher Scientific, Inc.) and adjusted to $1 \times 10^{6}$ cells/ml in DMEM. DMEM $(600 \mu 1)$, containing 10\% FBS was loaded in the lower chamber and $100 \mu \mathrm{l}$ resuspended cell solution was plated in the upper chamber. The plates were subsequently incubated for $16 \mathrm{~h}$ under normal conditions, after which non-invading or non-migrating cells on the upper surface of the membrane were removed from the chamber. The cells, which had invaded the lower surface of the membrane were fixed and stained with crystal violet (Sangon Biotech Co., Ltd.). The cells, which had migrated to the lower surface of the membrane were fixed and stained with eosin (ZSGB-Bio). The number of invading and migrating cells was calculated using a microscope (Olympus IX51; Olympus, Tokyo, Japan) at a magnification of x200 in five random fields. Three independent experiments were performed.

Gelatin zymography. Gelatin zymography assays were performed, as previously described (15), with minor modifications. Briefly, equal quantities of protein in the serum-free supernatant from transfected cells was diluted in $5 \mathrm{X}$ sample buffer $[10 \% \mathrm{SDS}(\mathrm{w} / \mathrm{v}) ; 0.05 \%$ bromophenol blue (w/v) and $0.25 \mathrm{M}$ Tris- $\mathrm{HCl}(\mathrm{pH}$ 6.8)] and incubated at room temperature for $10 \mathrm{~min}$. Subsequently, the proteins were separated by $10 \%$ SDS-PAGE, containing $1 \mathrm{mg} / \mathrm{ml}$ gelatin (Sigma-Aldrich, St. Louis, MO, USA). Each gel was subsequently transferred onto a clean glass container and washed in $2.5 \%$ Triton X-100 twice for $45 \mathrm{~min}$. The gels were incubated in a development buffer [50 mM Tris- $\mathrm{HCl} ; 150 \mathrm{mM} \mathrm{NaCl} ; 5$ $\mathrm{mM} \mathrm{CaCl}_{2} ; 1 \mu \mathrm{M} \mathrm{ZnCl} 2$ and $0.02 \% \mathrm{NaN}_{3}$, (pH 7.5)] for $16 \mathrm{~h}$ at $37^{\circ} \mathrm{C}$. Next, the gels were stained with $0.1 \%$ Coomassie brilliant blue R-250 (w/v; Sigma-Aldrich) in $45 \%$ methanol and $10 \%$ acetic acid for $1 \mathrm{~h}$ and were subsequently destained in $10 \%$ acetic acid for two 30 min washes. Clear bands were visualized in the areas where the gelatin was degraded. The bands were analyzed using densitometry with Image J software 1.46r (National Institute of Health, Bethesda, MD, USA). Three independent experiments were performed. 


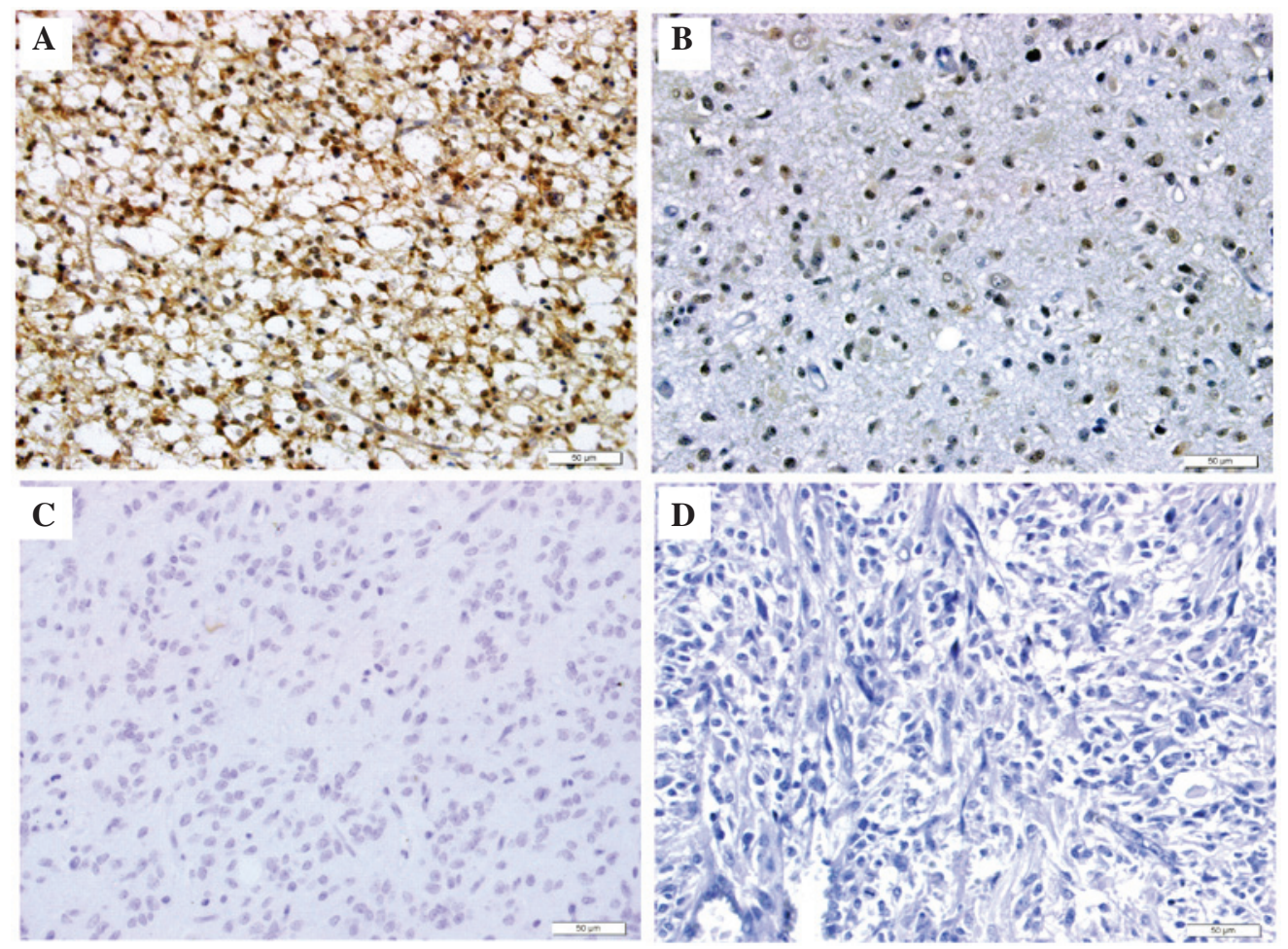

Figure 1. Immunohistochemical staining of KIF2A in glioma tissues. Positive staining for KIF2A was observed in the cytoplasm and/or nuclei of tumor cells, exhibiting a higher expression of KIF2A in (A) high-grade compared with (B) low-grade glioma tissues. (C) Negative staining for KIF2A in low-grade glioma tissues. (D) Negative staining for KIF2A in high-grade glioma tissues. 3,3'-Diaminobenzidine (brown) was used as a chromogen (scale bars=50 mm).

Flow cytometric analysisofapoptosis. An Annexin V-fluorescein isothiocyanate (FITC) and Propidium Iodide (PI) Apoptosis Detection kit (BestBio, Shanghai, China) was used to assess the apoptotic rate of the A172 cells pretreated with scrambled siRNA or KIF2A siRNA, according to the manufacturer's protocol. Briefly, A172 cells transfected with scrambled siRNA or KIF2A siRNA were seeded into 12-well plates and cultured under normal conditions for $48 \mathrm{~h}$. The cells were subsequently collected with trypsin (no EDTA), washed twice with cold PBS, resuspended with binding buffer and incubated with Annexin V-FITC and PI staining solution in the dark for $10 \mathrm{~min}$ at room temperature, and the apoptotic rate was immediately quantified using a FACSCalibur flow cytometer (BD Biosciences, San Jose, CA, USA). Three independent experiments were performed.

Statistical analysis. Statistical analysis was performed using SPSS 17.0 software (SPSS, Inc., Chicago, IL, USA) for Windows. The $\chi^{2}$ test was used to examine the correlation between the expression of KIF2A and various clinicopathological parameters. Student's t-test or Mann-Whitney U test was used for statistical analyses. $\mathrm{P}<0.05$ was considered to indicate a statistically significant difference.

\section{Results}

$m R N A$ and protein expression levels of KIF $2 A$ were significantly higher in grade III-IV compared with those in grade I-II glioma tissues. A summary of the patient clinicopathological features is provided in Table I. The present study evaluated the mRNA and protein expression levels of KIF2A in fresh glioma tissues by RT-qPCR and IHC, respectively. The mRNA expression levels of KIF2A were demonstrated to be significantly higher in grade III-IV glioma tissues compared with those in grade I-II glioma tissues (Table I; P<0.05). IHC analysis results showed that KIF2A was predominantly localized in the nucleus and cytoplasm of cancer cells (Fig. 1). Among the grade III-IV glioma specimens, 14 (40.0\%) were positively stained and $6(17.14 \%)$ revealed no staining. Among the grade I-II glioma specimens, 5 (14.29\%) were positively stained and $10(28.57 \%)$ revealed no staining. A significant increase in the protein expression of KIF2A was observed in grade III-IV glioma compared with grade I-II glioma $(\mathrm{P}<0.05$; Table I). Representative images are shown in Fig. 1.

Correlation between the expression of KIF $2 A$ and clinical parameters. The $\chi^{2}$ test results revealed that the expression of KIF2A correlated closely with the World Health Organization grade of glioma (Table I; $\mathrm{P}<0.05$ ). No significant correlation was observed between the expression of KIF2A, and patient age, gender and tumor location or size (Table I; P>0.05).

KIF2A knockdown significantly inhibits proliferation and induces apoptosis in A172 cells. To identify the functions of KIF2A in malignant glioma cells, the expression of KIF2A in the A172 and U251 glioma cell lines was analyzed. RT-qPCR results revealed that the mRNA expression of KIF2A was significantly higher in A172 cells compared with in U251 cells (Fig. 2A; P<0.001). The A172 cells were therefore selected for the in vitro experiments in the present study. Briefly, the human glioma cell lines, A172 and U251, were passaged to obtain the appropriate cells. The A172 and U251 cells were 
A

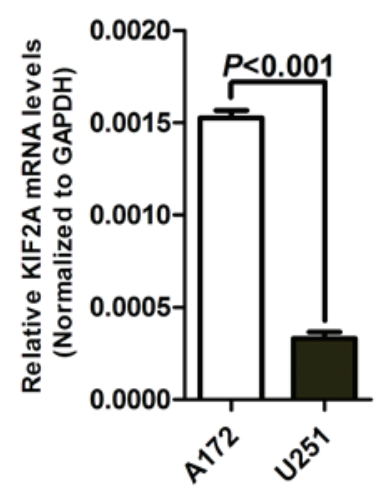

B

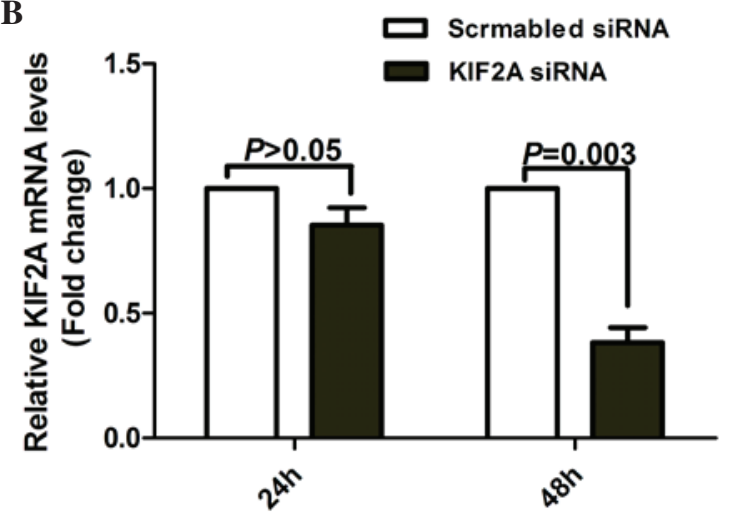

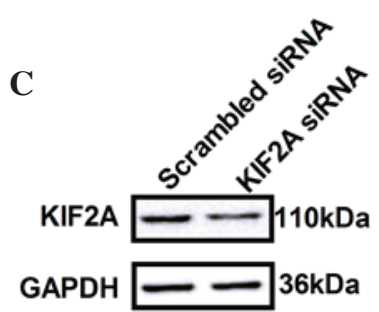

Figure 2. Evaluation of the expression of KIF2A and confirmation of RNA interference targeting KIF2A in glioma cells. (A) RT-qPCR was performed to determine the mRNA expression of KIF2A in glioma cell lines. The expression of KIF2A in A172 cells was demonstrated to be significantly higher compared with that in U251 cells ( $\mathrm{P}<0.001$; Student's t-test). (B) Confirmation of RNA interference against KIF2A in A172 cells by RT-qPCR (P $>0.05$ and P=0.003; Student's t-test). (C) Confirmation of RNA interference against KIF2A in A172 cells by western blot analysis. RT-qPCR, reverse transcription-quantitative polymerase chain reaction.

A

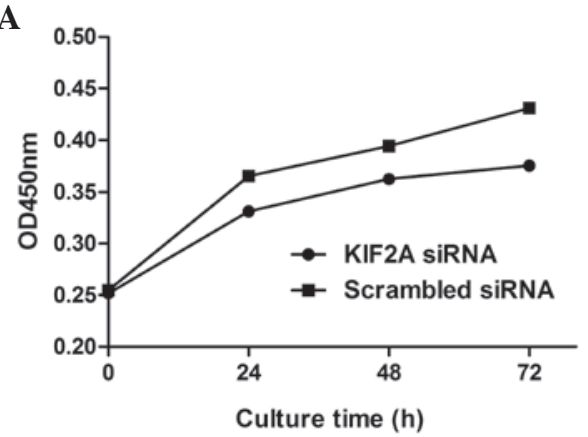

B

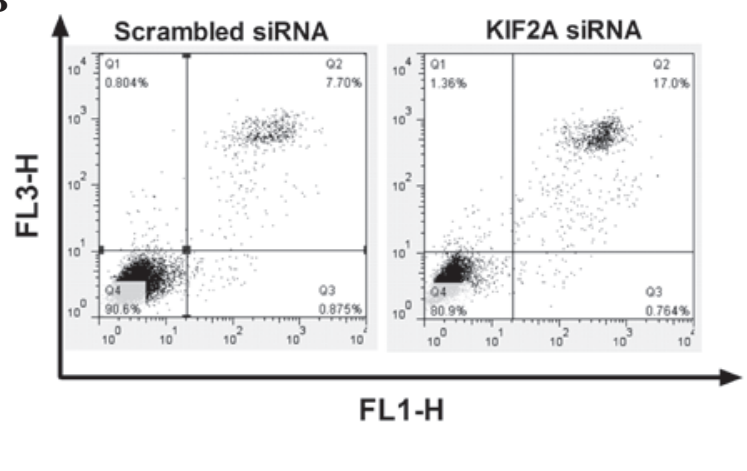

C

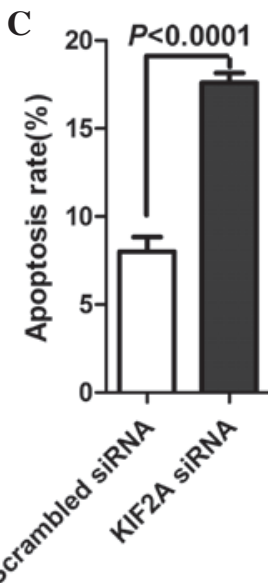

Figure 3. KIF2A knockdown significantly inhibited proliferation and induced apoptosis in A172 cells. (A) A CCK-8 assay was performed to determine the proliferation of A172 cells in response to the indicated treatments ( $\mathrm{n}=5$; data are presented as the mean). (B) Representative image of flow cytometric analysis to show the proportions of apoptotic cells in A172 cells transfected with the indicated siRNAs. (C) Bar graphs showing the ratios of apoptotic cells in A172 cells transfected with the indicated siRNAs ( $\mathrm{P}<0.0001$; Student's t-test). OD, optical density; siRNA, small-interfering RNA.

grown in DMEM supplemented with $10 \%$ heat-inactivated FBS at $37^{\circ} \mathrm{C}$ in $5 \% \mathrm{CO}_{2}$. Total RNA was extracted from the A172 and U251 cells, then reverse transcribed into cDNA and amplified by RT-qPCR. The A172 cell line then underwent further study. Compared with the scrambled siRNA group, A172 cells transfected with KIF2A siRNA exhibited significantly downregulated mRNA expression of KIF2A in a time-dependent manner (Fig. 2B). Consistent with the mRNA results, the protein expression of KIF2A was also lower in A172 cells transfected with KIF2A siRNA for $48 \mathrm{~h}$, compared with the scrambled siRNA group (Fig. 2C). The above results suggested that the specific siRNA targeting KIF2A significantly decreased the mRNA and protein expression levels of KIF2A.

The CCK-8 assay was used to investigate the effect of KIF2A on the proliferation of A172 cells. KIF2A knockdown inhibited A172 cell proliferation in a time-dependent manner (Fig. 3A). To assess whether the decreased cell number was due to apoptosis, induced by KIF2A siRNA, apoptosis in A172 cells following siRNA transfection was examined. As shown in Fig. 3B and $\mathrm{C}$, a higher number of apoptotic cells were observed among the A172 cells transfected with KIF2A siRNA, compared with the control cells $(\mathrm{P}<0.0001)$. The above data suggested that KIF2A may be important in the proliferation and apoptosis of glioma cells.

KIF2A knockdown significantly inhibited the invasion and migration capacities of A172 cells by regulating the activity and expression of $M M P-2$. Matrigel cell invasion and Transwell cell migration models were performed to analyze the effect of KIF2A expression on the invasion and migration capacities of A172 cells. Compared with the cells transfected with scrambled siRNA, the invasion and migration capacities of A172 cells were significantly inhibited by KIF2A knockdown (Fig. 4A and $\mathrm{B} ; \mathrm{P}<0.001$ and $\mathrm{P}=0.001$, respectively).

Matrix metalloproteinases (MMPs) are a group of peptidases involved in the degradation of the extracellular matrix, and accumulated data have suggested that MMPs contribute to the invasion and migration processes of glioma cells (16). Gelatin zymography was performed to analyze 


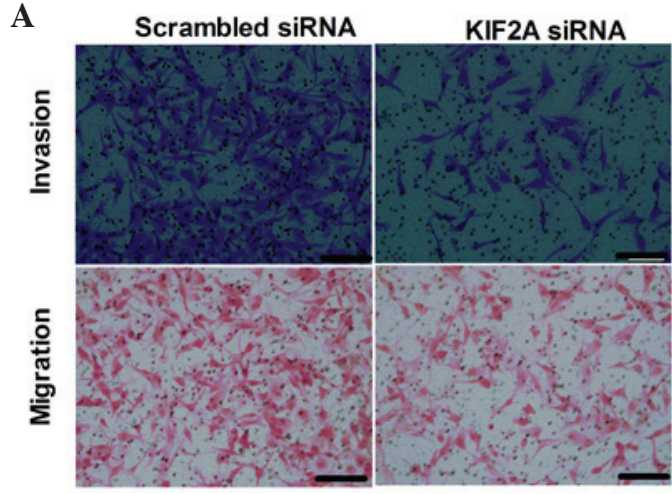

D

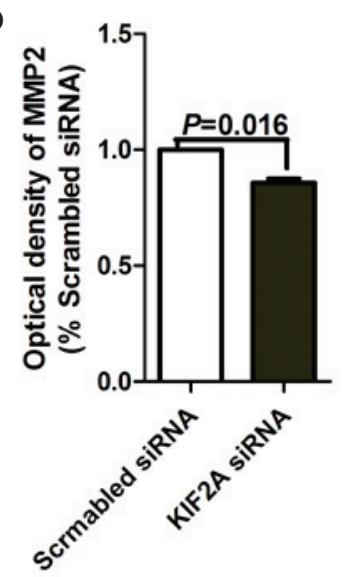

B

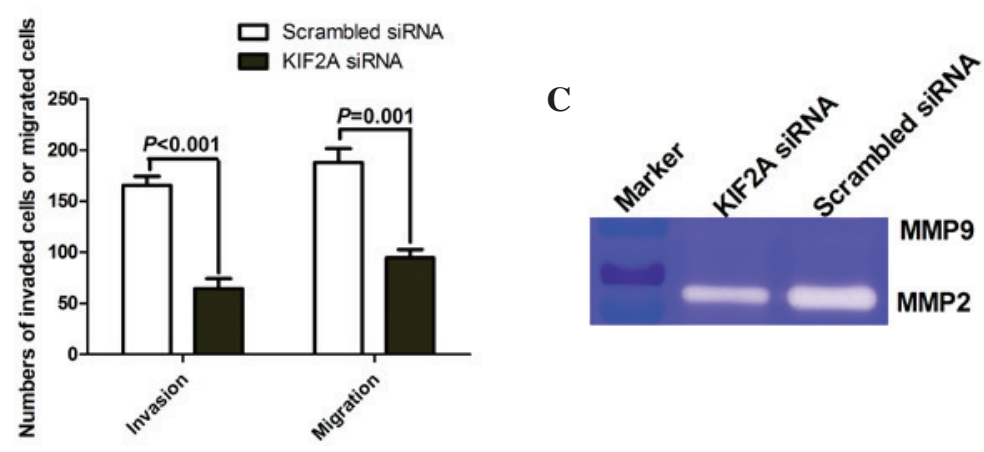

$\mathbf{F}$

$\mathbf{E}$

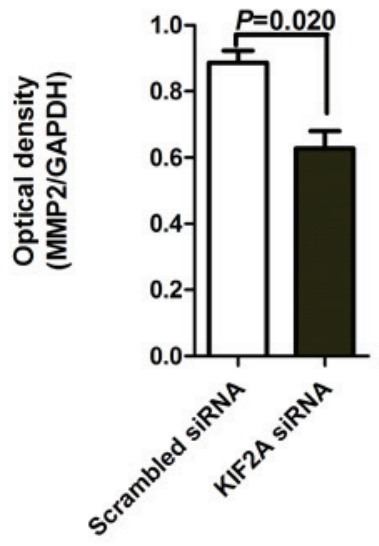

Figure 4. KIF2A siRNA inhibits the ability of A172 cells to invade and migrate. (A) Representative images of invasion and migration by A172 cells treated with the indicated siRNAs, as determined by Matrigel invasion and Transwell migration assays (scale bars, $100 \mu \mathrm{m}$ ). (B) Bar graphs showing the effects of KIF2A knockdown on invasion and migration in $\mathrm{A} 172$ cells $(\mathrm{n}=3 ; \mathrm{P}<0.001$ and $\mathrm{P}=0.001$; Student's t-test). (C) Zymographic analysis showing MMP-2/9 activity in serum-free medium from A172 cells transfected with the indicated siRNAs. (D) Statistical analysis of the representative zymographic results demonstrated in (C) where $\mathrm{n}=3$ ( $\mathrm{P}=0.016$; Student's t-test). (E) Western blot analysis showing the expression of MMP-2 in A172 cells transfected with the indicated siRNAs. GAPDH was used as an internal control. (F) Statistical analysis of the western blotting results, for which a representative blot is shown in (E) (n=3; $\mathrm{P}=0.020$, Student's t-test). siRNA, small interfering RNA.

the gelatinolytic activities of MMP-2 and MMP-9 in the supernatants of A172 cells transfected with KIF2A siRNA or scrambled siRNA. The results (Fig. 4C) and statistical analysis (Fig. 4D) demonstrated that KIF2A siRNA significantly decreased MMP-2 activity in the supernatant of A172 cells when compared with the scrambled siRNA $(\mathrm{P}=0.016)$. By contrast, MMP-9 activity was barely detected in the supernatant of A172 cells transfected with either indicated siRNA. Western blot analysis further demonstrated that KIF2A silencing significantly reduced the expression of MMP-2 ( $\mathrm{P}=0.020$; Fig. 4E and F), and MMP-9 expression levels were below detection limits (data not shown).

\section{Discussion}

The aim of the present study was to evaluate the expression of KIF2A in glioma tissues, assess the association between the expression of KIF2A and clinical parameters, as well as identify the functions of KIF2A in malignant glioma cells.

KIF2A, a member of the kinesin-13 family, was first cloned from the murine central nervous system by degenerate PCR in 1992 (17), and is said to act as the key factor in human neuronal diseases $(17,18)$. KIF2A has been reported to specifically localize to centrosomes and spindle poles (19), and is important in both bipolar spindle assembly and chromosome movement during mitosis $(19,20)$. A previous study reported that errors in the process of mitosis may result in numerous defects in daughter cells, thereby leading to carcinogenesis (21). Other previous studies on malignant tumor types have demonstrated that the overexpression of KIF2A is associated with tumor progression, including that of SCCOT and breast cancer $(10,11)$; however, the expression of KIF2A and its correlation with various clinical parameters in glioma remain unclear.

In the present study, the findings of RT-qPCR and IHC analysis demonstrated that the mRNA and protein expression levels of KIF2A were significantly higher in grade III-IV glioma tissues compared with those in grade I-II glioma tissues, and that the expression of KIF2A was notably correlated with glioma grade (Table I). This result was consistent with previous studies performed on breast cancer and SCCOT $(10,11)$, and suggested that KIF2A may be involved in the regulation of glioma tumor progression.

Previous studies have reported that the knockdown of KIF2A in somatic or cancer cells can lead to a marked increase in monopolar spindles $(9,20)$. Therefore, KIF2A may be important in the proliferation and apoptosis of those cells. In the present study, it was revealed that KIF2A knockdown significantly inhibited the proliferation of A172 cells (Fig. 3A), which supported the findings of previous studies on breast 
cancer and SCCOT. It was further demonstrated that KIF2A knockdown significantly induced apoptosis in A172 cells. A previous study also reported that KIF2A has a central role in the apoptosis of malignant cells (22). The precise mechanisms by which KIF2A regulates the proliferation and apoptosis of glioma cells remain to be elucidated.

Cellular morphology is supported by the cytoskeleton, and cytoskeletal reorganizations have important effects on the migration of neoplastic cells (12). Microtubules (MTs) are fundamental components of the cytoskeleton in eukaryotic cells. Accumulating evidence has revealed that decreases in MTs and MT depolymerization are important in the invasion and migration capacities of malignant tumors $(23,24)$. Certain previous studies have proposed that KIF2A has microtubule-depolymerizing activity $(9,25)$, which means that KIF2A may be involved in the movement of cells. In the present study, it was shown that KIF2A knockdown significantly inhibited the invasion and migration capacities of A172 cells (Fig. 4A and B); similar results have also been demonstrated in previous studies on breast cancer and SCCOT $(10,11)$. The present study further identified why KIF2A knockdown regulates the invasion and migration capacities of A172 cells. Due to the important role of MMPs in the invasion and migration of glioma cells (16), the activity and expression of MMP-2/9 in A172 cells transfected with KIF2A/scramble siRNA were analyzed by gelatin zymography and western blot analysis. The results revealed that KIF2A significantly inhibited the activity and expression of MMP-2 in A172 cells, however, the activity and expression of MMP-9 were below the detection limit (Fig. 4C-F). The precise mechanism by which KIF2A regulates the expression and function of MMP-2 remains to be elucidated.

In conclusion, the findings of the present study demonstrated that the expression of KIF2A was upregulated in grade III-IV glioma tissues compared with that in grade I-II glioma tissues, and that it was markedly correlated with glioma grade. Furthermore, it was revealed that KIF2A knockdown significantly inhibited proliferation and induced apoptosis in A172 cells, as well as significantly inhibited the invasion and migration capacities of A172 cells by regulating the activity and expression of MMP-2. These results suggested that KIF2A has a central role in glioma development and that the inhibition of KIF2A expression may prove to be a promising target in the control of glioma.

\section{Acknowledgements}

The present study was supported by grants from the National Natural Science Foundation of China (grant nos. 31470885 and 31270971) and the Natural Science Foundation of Science and Technology Department of Liaoning Province (grant no. 2014022020).

\section{References}

1. Ohgaki H and Kleihues P: Epidemiology and etiology of gliomas. Acta Neuropathol 109: 93-108, 2005.

2. Maher EA, Furnari FB, Bachoo RM, Rowitch DH, Louis DN, Cavenee WK and DePinho RA: Malignant glioma: Genetics and biology of a grave matter. Genes Dev 15: 1311-1333, 2001.

3. Miki H, Okada Y and Hirokawa N: Analysis of the kinesin superfamily: Insights into structure and function. Trends Cell Biol 15: 467-476, 2005.
4. Lukong KE and Richard S: Breast tumor kinase BRK requires kinesin-2 subunit KAP3A in modulation of cell migration. Cell Signal 20: 432-442, 2008.

5. Corson TW and Gallie BL: KIF14 mRNA expression is a predictor of grade and outcome in breast cancer. Int J Cancer 119: 1088-1094, 2006.

6. Minakawa Y, Kasamatsu A, Koike H, Higo M, Nakashima D, Kouzu Y, Sakamoto Y, Ogawara K, Shiiba M, Tanzawa H and Uzawa K: Kinesin family member 4A: A potential predictor for progression of human oral cancer. PLoS One 8: e85951, 2013

7. Bie L, Zhao G, Wang YP and Zhang B: Kinesin family member $2 \mathrm{C}(\mathrm{KIF} 2 \mathrm{C} / \mathrm{MCAK})$ is a novel marker for prognosis in human gliomas. Clin Neurol Neurosurg 114: 356-360, 2012

8. Taniwaki M, Takano A, Ishikawa N, Yasui W, Inai K, Nishimura H, Tsuchiya E, Kohno N, Nakamura Y and Daigo Y: Activation of KIF4A as a prognostic biomarker and therapeutic target for lung cancer. Clin Cancer Res 13: 6624-6631, 2007.

9. Ganem NJ and Compton DA: The KinI kinesin Kif2a is required for bipolar spindle assembly through a functional relationship with MCAK. J Cell Biol 166: 473-478, 2004.

10. Wang CQ, Qu X, Zhang XY, Zhou CJ, Liu GX, Dong ZQ, Wei FC and Sun SZ: Overexpression of Kif2a promotes the progression and metastasis of squamous cell carcinoma of the oral tongue. Oral Oncol 46: 65-69, 2010

11. Wang J, Ma S, Ma R, Qu X, Liu W, Lv C, Zhao S and Gong Y: KIF2A silencing inhibits the proliferation and migration of breast cancer cells and correlates with unfavorable prognosis in breast cancer. BMC Cancer 14: 461, 2014

12. Louis DN, Ohgaki H, Wiestler OD, Cavenee WK (eds): WHO classification of tumours of the central nervous system. IV edition. IARC Press, Lyon, pp8-57, 2007.

13. Wang N, Feng Y, Wang Q, Liu S, Xiang L, Sun M, Zhang X, Liu G, Qu X and Wei F: Neutrophils Infiltration in the tongue squamous cell carcinoma and its correlation with CEACAM1 expression on tumor cells. PLoS One 9: e89991, 2014.

14. Kawai H, Ishii A, Washiya K, Konno T, Kon H, Yamaya C, Ono I, Minamiya Y and Ogawa J: Estrogen receptor alpha and beta are prognostic factors in non-small cell lung cancer. Clin Cancer Res 11: 5084-5089, 2005.

15. Wang H, Cheng H, Shao Q, Dong Z, Xie Q, Zhao L, Wang Q, Kong B and Qu X: Leptin-promoted human extravillous trophoblast invasion is MMP14 dependent and requires the cross talk between Notch1 and PI3K/Akt signaling. Biol Reprod 90: 78, 2014.

16. Nakada M, Okada Y and Yamashita J: The role of matrix metalloproteinases in glioma invasion. Front Biosci 8: e261-e269, 2003.

17. Aizawa H, Sekine Y, Takemura R, Zhang Z, Nangaku M and Hirokawa N: Kinesin family in murine central nervous system. J Cell Biol 119: 1287-1296, 1992.

18. Poirier K, Lebrun N, Broix L, Tian G, Saillour Y, Boscheron C, Parrini E, Valence S, Pierre BS, Oger M, et al: Mutations in TUBG1, DYNC1H1, KIF5C and KIF2A cause malformations of cortical development and microcephaly. Nat Genet 45: 639-647, 2013.

19. Ganem NJ, Upton K and Compton DA: Efficient mitosis in human cells lacking poleward microtubule flux. Curr Biol 15: 1827-1832, 2005

20. Zhu C, Zhao J, Bibikova M, Leverson JD, Bossy-Wetzel E, Fan JB, Abraham RT and Jiang W: Functional analysis of human microtubule-based motor proteins, the kinesins and dyneins, in mitosis/cytokinesis using RNA interference. Mol Biol Cell 16: 3187-3199, 2005.

21. Weaver BA and Cleveland DW: Decoding the links between mitosis, cancer and chemotherapy: The mitotic checkpoint, adaptation and cell death. Cancer Cell 8: 7-12, 2005.

22. Wang K, Lin C, Wang C, Shao Q, Gao W, Song B, Wang L, Song X, Qu X and Wei F: Silencing Kif2a induces apoptosis in squamous cell carcinoma of the oral tongue through inhibition of the PI3K/Akt signaling pathway. Mol Med Rep 9: 273-278, 2014.

23. Fink-Puches R, Hofmann-Wellenhof R, Smolle J, Helige C and Kerl H: Cytoplasmic microtubules in two different mouse melanoma cell lines: A qualitative and quantitative analysis using confocal laser scanning microscopy and computer-assisted image analysis. J Cutan Pathol 24: 350-355, 1997.

24. Watanabe T, Noritake J and Kaibuchi K: Regulation of microtubules in cell migration. Trends Cell Biol 15: 76-83, 2005.

25 . Niwa $S$ : Kinesin superfamily proteins and the regulation of microtubule dynamics in morphogenesis. Anat Sci Int 90: 1-6, 2015. 